\title{
La recherche en éducation environnementale - Interrogations au Sahel
}

\section{Moussa Batchily Ba}

\author{
(2) OpenEdition \\ Journals \\ Édition électronique \\ URL : https://journals.openedition.org/ere/7278 \\ DOI : $10.4000 /$ ere. 7278 \\ ISSN : 2561-2271 \\ Éditeur \\ Centr'ERE
}

Référence électronique

Moussa Batchily $\mathrm{Ba}$, « La recherche en éducation environnementale - Interrogations au Sahel », Éducation relative à l'environnement [En ligne], Volume 1 | 1999, mis en ligne le 15 septembre 1999, consulté le 28 mai 2021. URL : http://journals.openedition.org/ere/7278 ; DOI : https://doi.org/ 10.4000/ere. 7278

Ce document a été généré automatiquement le 28 mai 2021. 


\title{
La recherche en éducation environnementale - Interrogations au Sahel
}

\author{
Moussa Batchily Ba
}

1 Si de façon globale, les défis environnementaux interpellent la communauté internationale, il existe des régions de notre planète qui sont dans des situations de rupture particulièrement graves. Au nombre de celles-ci, le Sahel: désertification, croissance démographique accélérée, pollution, manque d'hygiène, assainissements déficients, mauvaises évacuations des déchets et des eaux usées, inaccessibilité aux ressources naturelles, particulièrement en eau et en bois de chauffage, pauvreté et déficience des services sociaux (santé - éducation).

2 La vision de l'environnement de l'après RIO implique une éthique nouvelle de l'environnement qui serait synonyme d'une responsabilisation intrinsèque de chaque citoyen de la planète Terre (Beauchamp, 1993). Un tel engagement doit être sous-tendu par un processus éducatif total et auto-entretenu. C'est le sens du chapitre 36 de l'Agenda 21 (CNUED, 1992).

3 Précédant ou s'inscrivant dans le sillage de Rio, un vaste mouvement s'est développé au Sahel en faveur de l'éducation environnementale (EE) autant en contexte non formel que formel. Toutes les actions entreprises en ce sens sont considérées comme des terrains d'expérimentation ouverts qui militent pour une exigence forte: celle de contribuer à l'avènement d'un développement durable et équitable. Cette exigence se traduit par des défis à relever.

4 Un premier défi est de mettre en œuvre des projets qui profitent aux générations actuelles et futures. Un second défi est de rendre ces projets bénéfiques pour la région sahélienne, où se développent des stratégies de survie. Un troisième défi est l'impact durable des dits projets tout en les inscrivant dans le cadre de stratégies et de politiques d'EE affichées. 
5 En effet, expérimenter et généraliser des innovations sans validation scientifique et en dehors d'une optique de durabilité comporte une hypothèque grave pour la jeunesse sahélienne et pour les populations désemparées devant l'école d'aujourd'hui.

Pour répondre à ce premier niveau structurant, différents acteurs ont senti la nécessité de faire de la recherche-action en vue d'apporter des réponses satisfaisantes quant au bien-fondé des options envisagées ou des hypothèses formulées.

7 Dans la littérature relative à l'EE, il apparaît que des efforts de réflexion ont été faits dans les domaines suivants : la définition des concepts, les approches méthodologiques, l'articulation entre formel et non formel, l'évaluation de programmes, les questions transversales portant sur la mobilisation sociale, la question du genre et de la pauvreté, la prise en compte du savoir local, les politiques et stratégies. Toutes ces questions doivent être clarifiées en vue de favoriser la vulgarisation de l'EE.

Mais quels sont les centres d'intérêt au Sahel et dans quels termes se posent ces questions?

\section{Les interrogations}

9 Une première interrogation est la variation sensible (parfois importante) dans la définition explicite du concept d'EE (Sauvé, 1991). Celle-ci nécessite une clarification conceptuelle pouvant permettre aux pays sahéliens d'être fixés sur le contenu, les processus et modalités de mise en œuvre de programmes et de politiques d'EE aptes à répondre aux besoins des populations.

10 Un second centre d'intérêt concerne les politiques et stratégies en EE. Le constat premier est que sous "l'effet de mode", des actions, louables par ailleurs, se sont développées sans que soient garanties leurs conditions d'efficacité et de durabilité. Face à cela, des initiatives sont en cours pour élaborer des cadres de référence. Dans ce contexte, comment assurer un processus participatif au regard des intérêts des groupes, pour que les acteurs endossent la pérennité des activités? Comment articuler cette préoccupation avec les engagements liés aux conventions internationales (désertification, biodiversité, changement climatique et autres)?

11 Un autre centre d'intérêt a trait aux questions relatives à l'éducation et au système éducatif. Il convient de se pencher d'abord sur les approches méthodologiques y compris les approches pédagogiques et outils didactiques. Quelles approches et quels outils apparaissent appropriés en EE dans la mesure où il s'agit d'appréhender le sociosystème et l'écosystème dans leurs particularités, leurs dynamiques propres, et de percevoir les interactions entre leurs différents éléments? La recherche pourrait s'intéresser aux liens entre les fondements de l'éducation (la sociologie, l'économie, les politiques, la question du genre, etc.) et l'EE.

Ensuite, il faut aborder la question de l'évaluation. Pour prétendre à une pérennisation du comportement positif envers l'environnement, il y a une nécessité de certification des actions en EE. Dès lors, quelle stratégie d'évaluation des apprentissages doit-on adopter? Comment assurer la cohérence avec le type d'enseignement privilégié ? Fautil mettre l'accent sur une dimension formative ou sur les tests sommatifs? Quel processus éducatif ou modèle permet une évaluation appropriée des actions en EE ? Les résultats de la recherche permettraient d'éclairer les éducateurs, les populations, les 
partenaires et les décideurs sur ces différents questionnements relatifs aux méthodologies et aux stratégies à mettre en œuvre.

Enfin, il paraît légitime de soulever d'autres aspects pédagogiques qui réfèrent entre autres à une modélisation de systèmes d'enseignement ou d'apprentissage (modèles éducatifs formels ou non formels) avec des buts définis et partagés, des stratégies pédagogiques, des outils et des procédures de certification (Giolitto et Clary, 1994). L'aspect pédagogique revêt une importance capitale dans la mesure où l'EE vise davantage le développement de compétences que l'acquisition de connaissances, cette dernière caractérisant le système éducatif sahélien actuel où le savoir est morcelé. Il paraît également judicieux d'évoquer des aspects externes à la situation d'apprentissage et liés en grande partie aux politiques éducatives et sociales.

14 Par ailleurs, dans un contexte de crise des systèmes éducatifs au Sahel, l'EE ne devraitelle pas constituer une passerelle entre le système éducatif formel et le système éducatif non formel? Un programme d'EE ne doit-il pas permettre de créer une synergie entre les différentes composantes du système social (hommes, femmes, enfants et jeunes scolarisés, déscolarisés et ceux non scolarisés) en relation avec les écosystèmes et non favoriser une juxtaposition entre elles? Tenant compte de l'organisation socio-éducative traditionnelle, la recherche doit permettre d'identifier les types de stratégies qui s'adaptent le mieux à la mise en œuvre d'une EE pour une société globale.

15 Afin de maximiser les chances d'aboutir à la finalité à laquelle postule l'EE, à savoir le développement d'attitudes et de comportements favorables à une gestion rationnelle de l'environnement, les recherches doivent permettre de savoir s'il faut une combinaison des différentes approches connues, une complémentarité ou une exclusivité, voire une innovation totale dans ce domaine.

16 À ces questions relatives à l'éducation viennent se joindre d'autres questions liées à la gestion de l'environnement pour former le couple éducation et environnement. Il importe de prendre en compte le lien entre la dégradation de l'environnement et la pauvreté qui fait appel à des stratégies de survie. Dans ce contexte, comment établir une collaboration étroite entre les communautés et les groupes porteurs comme les femmes et les enfants (qui sont les plus sérieusement affectés dans la spirale de la pauvreté) pour une gestion durable des ressources?

17 Les actions quotidiennes des enfants et des femmes et leur participation à la recherche de solutions nouvelles et novatrices dans la gestion de l'environnement représentent des jalons importants dans la responsabilisation progressive de tout citoyen et dans la pérennisation des acquis. L'EE ne devrait-elle pas offrir de nouvelles opportunités d'apprentissages fonctionnels générateurs de revenus?

18 Dans le même ordre d'idées, on doit reconnaître que la création des conditions favorables à un dialogue fécond entre le savoir environnemental traditionnel et le savoir scientifique moderne, entre enfants (filles et garçons), parents (femmes et hommes), praticiens de l'éducation, ONG, toutes autres institutions gouvernementales et non gouvernementales permettraient de mieux promouvoir la mobilisation sociale pour l'EE (Eva, 1992). Comment établir à cet effet des alliances stratégiques pérennes? 


\section{Conclusion}

19 Au bilan, on perçoit qu'il y a bien une vision d'ensemble qu'il convient d'adopter au risque de retomber dans des approches parcellaires. Cela est d'autant plus important que la prise en compte de l'EE va induire une révision des procédures d'apprentissage, voire une révision profonde des systèmes éducationnels actuels, cela au regard des objectifs liés à cette innovation.

Un diagnostic permettrait tout à la fois d'établir le profil des types de chercheurs requis et les besoins de recherche pour faire avancer l'EE. Il s'agit entre autres de cerner tout ce qui peut toucher à la conceptualisation de l'EE : comment est-elle perçue par les différents acteurs? Qu'en est-il des méthodologies, des contenus? On entrevoit ici des recherches concernant la «didactique des disciplines» ou encore, la pédagogie générale (Ba, 1996).

21 Afin d'assurer un plaidoyer pour l'environnement, il importe d'entamer des recherches sur les politiques, leurs mesures d'accompagnement et leurs éléments facilitateurs de projets pédagogiques.

Dans un effort de synergie régionale, les différentes interrogations passées ici en revue ont permis de démarrer un programme régional de recherche en éducation environnementale au Sahel (PREES, financé par le Centre de recherches pour le développement international du Canada) qui offre la possibilité aux chercheurs de développer des thèmes de recherche sous la responsabilité d'un comité scientifique $(\mathrm{Ba}$, 1993; Ba et Camilla, 1996). Les résultats des recherches et des projets d'éducation environnementale au Sahel donneront lieu à des publications et à une présentation lors d'un Forum international sur l'EE envisagé pour novembre 1999 au Sahel.

\section{BIBLIOGRAPHIE}

Ba, M. (1993). Politiques environnementales : Institutions et structures impliquées dans la gestion de l'environnement au Sahel. Dakar : CRDI.

Ba, M.B. (1996). Proposition de recherche soumise au CRDI. Bamako : Institut du Sahel.

Ba, M.B. et Camilla, T. (1996). Le programme de formation-information sur l'environnement. Communication présentée dans le cadre de l'Atelier sur le développement des capacités dans le domaine de l'environnement, 4-6 décembre 1996, Rome. OCDE.

Beauchamp, A. (1993). Introduction à l'éthique de l'environnement. Montréal : Éditions Paulines.

Conférence des Nations unies sur l'Environnement et le Développement (CNUED). (1992). Agenda 21. Programme d'action pour le développement durable : Déclaration de Rio sur l'environnement et le développement. New York: ONU.

Eva, M.R. (1992). Bringing gender and environnement into the curriculum : some african examples. Nairobi, Kenya : IDRC. 
Giolitto, P. et Clary, M. (1994). Éduquer à l'environnement. Paris : Édition Hachette Éducation.

Sauvé, L. (1991). Essai de typologie des approches concernant l'objet d'apprentissage en éducation relative à l'environnement. In JIES (dir.), Actes des XIIIes Journées internationales sur la communication, l'éducation et la culture scientifique et industrielle. École et médias face aux défis de l'environnement. Chamonix, France : JIES. p. 51-55.

\section{AUTEUR}

\section{MOUSSA BATCHILY BA}

Moussa Batchily Ba est historien de formation et enseignant. Il coordonne pour l'Institut du Sahel, un programme régional d'éducation environnementale qui comprend trois volets : un volet pour le primaire (Programme de formation-information sur l'environnement - PFIE) soutenu par la Commission Européenne, un volet pour le secondaire (Programme sahélien niveau secondaire - PSE2) soutenu par l'UNSO et un volet pour la recherche (Programme régional de recherche en éducation environnementale au Sahel - PREES) appuyé par le Centre de recherches pour le développement international (CRDI, Canada). 